\title{
3 ENTREPRENEURE IN LÄNDLICHEN RÄUMEN - SCHNITTSTELLEN IN NETZWERKEN?
}

Gesine TUITJER, Thünen-Institut für Ländliche Räume, Braunschweig

\section{INHALT}

3.1 Einleitung

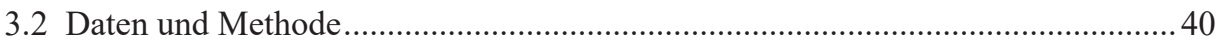

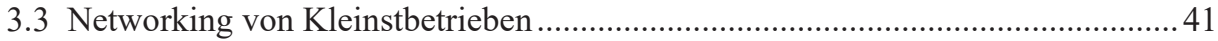

3.3.1 „Verstehen“ und „Nicht-Verstehen“ als Muster des Netzwerkaufbaus............... 42

3.3.2 Ressourcen sichern und erschließen durch lokale Netzwerke ........................... 44

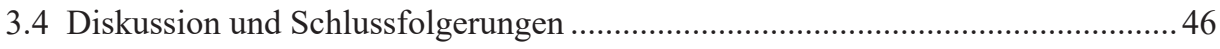

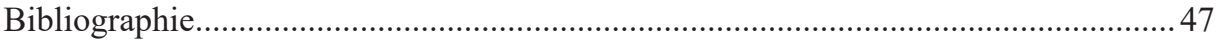

Der vorliegende Beitrag beschäftigt sich mit dem Netzwerk- und Innovationsverhalten von Kleinstbetrieben in ländlichen Räumen. Der Beitrag verortet sich in der Diskussion um das Spannungsfeld zwischen der Verwurzelung am betrieblichen Standort und dem Aufbau räumlich weitreichender Netzwerke als betriebliche Strategien, die die periphere Lage kompensieren können. Es zeigt sich, dass die Entrepreneure vor allem symbolisches Wissen aus ihren überregionalen Netzwerken beziehen. Um dieses Wissen jedoch in Produktinnovationen umsetzen zu können, sind die lokalen Beziehungen entscheidend, denn über diese werden vor allem Rohstoffe und Verarbeitungswege erschlossen. Hierbei illustriert der Text die hohe Selektivität der Beziehungen, in denen die räumliche Dimension von Nähe nur eine von vielen ist. So findet ein Netzwerkaufbau - auch über weite räumliche Distanzen hinweg - primär unter ,Gleichen “ statt. Trotz eingeschränkter Übertragbarkeit werfen die Ergebnisse die Frage auf, inwiefern Entrepreneure daher Schnittstellen zwischen unterschiedlichen Netzwerken einnehmen können. 


\subsection{Einleitung}

Entrepreneurship wird eine große Bedeutung in der endogenen Entwicklung, insbesondere auch von ländlichen Regionen, beigemessen (für viele: ATTERTON 2016). Für ländliche Räume bestehen dabei insbesondere Entwicklungspotenziale in der unternehmerischen Erschließung spezifischer regionaler Ressourcen. Dies sind beispielsweise natürlich vorkommende Rohstoffe, aber auch kulturelles Kapital wie regionale Spezialitäten oder auch eine attraktive Landschaft (RAY 1999; ANDERSON 2000). Auch das Unternehmertum per se wird als endogene Ressource verstanden, wenn die Bereitschaft sich über den Betrieb hinaus für die regionale Entwicklung einzusetzen genutzt oder aktiviert werden kann. Die vertrauensvollen Netzwerke, die Unternehmer (u.U.) aufbauen, können auch der Regionalentwicklung dienlich sein (LASCHEWSKI et al. 2002; BÜRCHER \& MAYER 2018). Insbesondere für Kleinstbetriebe in Nischen können betriebliche Interessen und die Entwicklung des Standortes (zum Beispiel zur touristischen Attraktion etc.) zusammenfallen (TREGEAR 2005). Gleichzeitig ist bekannt, dass gerade Kleinstbetriebe - wie sie häufig in ländlichen Räumen zu finden sind - eher seltener untereinander kooperieren oder in regionale Netzwerke eingebunden sind. BJøRKHAUG \& KVAM (2011) untersuchen staatlich geförderte Lebensmittelmanufakturen in ländlichen Räumen in Norwegen und stellen fest, dass viele der knapp 300 befragten Unternehmer kein Interesse daran haben, lokale Netzwerke aufzubauen bzw. diesen beizutreten. Auch fällt es ihnen schwer, geeignete Partner auf lokaler Ebene zu finden. KNEAFSEY et al. (2001) vermuten, dass die Bereitschaft zu Kooperationen auf der lokalen Ebene und mit dem Ziel der regionalen Entwicklung sehr von der betrieblichen Strategie abhängt. Basierend auf einer Fallstudie aus Wales zeigen sie, dass gerade Nischenprodukte keiner territorialen Vermarktungsstrategie bedürfen.

Zur Erschließung endogener Ressourcen, insbesondere aber zu ihrer Vermarktung, sind jedoch auch überregionale Verbindungen im weitesten Sinne notwendig oder zumindest förderlich. Beispielsweise sind regionale Spezialitäten oder Sehenswürdigkeiten gerade für Touristen interessant, die so für Kapitalzuflüsse in ländliche Räume sorgen. Mitunter braucht es auch den Blick von außen, um die regionalen Besonderheiten und endogenen Potenziale überhaupt zu erkennen. Dies kann als neo-endogener Entwicklungsansatz verstanden werden (BOSWORTH \& ATTERTON 2012). Unternehmern kommt vor diesem Hintergrund eine besondere Rolle als Schnittstelle zwischen lokalen Besonderheiten und Ressourcen und deren Erschließung durch überregionales Wissen zu. Die Verbindung zu nicht-lokalen Akteuren wurde in vielen Studien als förderlich für die betriebliche Entwicklung und die Innovationsfähigkeit von Betrieben in ländlichen Räumen erkannt (Huggins \& JoHnston 2009; RodríGueZ-Pose \& FitJAR 2013; DubOIS 2016, MüLLER \& KorSGAARD 2018). Dabei bleibt jedoch untererforscht, welcher Art diese Verbindungen sind bzw. was genau über translokale Netzwerke bezogen wird und wie genau das Zusammenspiel von lokalen und überregionalen Netzen aussieht. In der Literatur wird sowohl die Position vertreten, dass Betriebe in ländlichen Räume eine 
entweder-oder-Strategie verfolgen, d.h. entweder lokal eingebettet sind und endogene Ressourcen erschließen oder in translokale Beziehungen eingebunden sind (KORSGAARD et al. 2015a). Abhängig vom Grad der lokalen Einbettung haben sie auch komplementäre Vorteile in der Nutzung lokaler oder überregionaler Ressourcen (KALANTARIDES \& BIKA 2006; KorsGaARD et al. 2015b). Diese teils widersprüchlichen Beobachtungen sind durch die große Vielfalt der Entrepreneure und der jeweils untersuchten Betriebe in ländlichen Räumen zu erklären. Ferner spielt selbstverständlich auch der komplexe regionale Kontext eine Rolle in der Entwicklung der Betriebe. Zugewanderte Entrepreneure profitieren darüber hinaus häufig von einer besseren Ausbildung und Kapitalausstattung (AKGÜN et al. 2011). Im Gegensatz dazu finden sich auch Hinweise auf sich gegenseitig verstärkende positive Effekte interner und regions-externer Netzwerke (DuBoIs 2016). Im Rahmen der Diskussion um lokale Embeddedness und überregionale Vernetzung wird mitunter die räumliche Distanz zwischen Akteuren/Betrieben als per se sinnhafte Dimension bzw. als Annäherung an andere Arten von Nähe im Innovationsprozess und der Netzwerkentwicklung verstanden (Boschma \& Frenken 2010) und ist dadurch tendenziell überbewertet. Boschma (2005) führt neben geographischer Nähe sowohl organisationale und institutionelle Nähe als auch kognitive und soziale Nähe an. Die Definition organisationaler Nähe verläuft entlang der Frage, wie die Akteure in Verbindung stehen, ob sie beispielsweise in einer marktförmigen oder eher einem hierarchischen Verhältnis zueinander stehen. Institutionelle Nähe kann als der Rahmen verstanden werden, unter dem Akteure agieren. Dies sind bei betrieblichen Kooperationen beispielsweise branchenübliche Verfahren, Konventionen oder die Größe der Betriebe. Die Unterscheidung zwischen kognitiver Nähe und sozialer Nähe kann als der Unterschied zwischen (wissensbasiertem) „Verstehen“ und „Vertrauen“ zwischen zwei Personen erfasst werden. So kann beispielsweise Vertrauen die gegenseitige Kooperation erleichtern, auch wenn die Handlungen des Gegenübers nicht vollständig verstanden werden.

Vor diesem Hintergrund soll anhand einer Fallstudie illustriert werden, wie selektiv Kleinstunternehmer in peripheren Räumen ihre Netzwerke auf- und ausbauen und wie sie (mitunter) innovativ werden. Hierzu wird ein qualitativer, praxistheoretischer Zugang gewählt, mit dessen Hilfe die Handlungsmuster und Sinngebungsprozesse der beteiligten Akteure interpretativ rekonstruiert werden. Somit wird in diesem Beitrag ein soziologischer Blick auf räumlich verortetes unternehmerisches Handeln gelegt. Ein besonderes Augenmerk liegt dabei auf dem Netzwerkaufbau, der sich im Zuge der Produktentwicklung vollzieht, und auf den hier auftretenden Schwierigkeiten und Herausforderungen. 


\subsection{Daten und Methode}

Der vorliegende Beitrag beruht auf Interviewmaterial, das im Rahmen eines Förderprogrammes für ländliche Räume erhoben wurde. Das Programm hat unter anderem zum Ziel, innovative Ansätze der Wertschöpfung in ländlichen Räumen zu erproben. Die regionale Fallstudie beleuchtet den Nordosten Deutschlands, eine stark agrarisch geprägte Region. Die Landwirtschaft ist hier, anders als beispielsweise in weiten Teilen Mittel- und Süddeutschlands, geprägt durch eine ,industrielle“ Landwirtschaft, d.h. durch sehr große Agrarbetriebe, die aus den ehemaligen Landwirtschaftlichen Produktionsgenossenschaften (LPGs) der DDR hervorgegangen sind. Da diese LPGs bereits auf die vorhandene Agrarstruktur und die vorherrschenden Großbetriebe aufbauten, fehlen neben der kleinteiligen „bäuerlichen“ Landwirtschaft sowohl Verarbeitungsbetriebe als auch Handwerksbetriebe aus dem Lebensmittelsektor (SIEBERT \& LASCHEWSKI 2010). Diese besondere Wirtschaftsstruktur unterscheidet die Region von anderen „typisch ländlichen“ Regionen und nimmt Einfluss auf die Entwicklung der Kleinstbetriebe, wie noch zu zeigen sein wird. Die Region ist überdies eine der am dünnsten besiedelten in Deutschland mit ca. 70 Einwohnern pro Quadratkilometer. Die relative Nähe zu den Zentren Hamburg und Berlin sowie die überdurchschnittliche Sonnenscheindauer sorgen für eine große Rolle des Tourismus in der Region. Gleichzeitig befördert die touristische Attraktivität den Zuzug sogenannter „Lifestyle-Entrepreneure“ (TREgear 2003 \& 2005), die für diese Studie untersucht wurden. Damit wird ein UnternehmerInnentyp beschrieben, der sich durch die gleichzeitige hohe Orientierung sowohl an wirtschaftlichen Aspekten (wie Entwicklung des Betriebes) als auch an sozialen bzw. lokalen Belangen auszeichnet. Für diesen Typ spielen sowohl die Selbstbestimmtheit der selbstständigen Tätigkeit eine große Rolle als auch die Verbundenheit mit der Region. Die hier untersuchten EntrepreneurInnen sind überwiegend zugezogen. Sie bringen ausreichend Ressourcen mit, um sowohl die Gründung der Kleinstbetriebe zu finanzieren als auch sich für die ersten Jahre selbst zu finanzieren. Es ist somit von einer höheren Ressourcenausstattung auszugehen. Dies deckt sich mit anderen Studien zur höheren Ressourcenausstattung zugewanderter Entrepreneure in ländlichen Räumen (AKGÜN et al. 2011; KorSGAARD et al. 2015b). Auch können alle EntrepreneurInnen als ,Opportunity “-driven verstanden werden; sie sehen also eine große Chance in der Selbstständigkeit und ziehen diese Beschäftigung - bzw. diesen Lebensstil - bewusst einer abhängigen Beschäftigung vor. Die EntrepreneurInnen sind im mittleren Alter und gründen teilweise mit dem Partner gemeinsam. Sie verfügen überwiegend über 20 bis 30 Jahre Berufserfahrung. In der Untersuchungsregion haben sie Kleinstbetriebe gegründet, die mehrheitlich zwei bis zehn Angestellte haben und einen Jahresumsatz unter 500.000 $€$ generieren. Die im Rahmen dieses Beitrags untersuchten UnternehmerInnen haben überwiegend Produktinnovationen hervorgebracht. Dies wird hier verstanden als ein Produkt, das zumindest im regionalen Markt neu ist. Typisch für ländliche Räume handelt es sich außerdem um Nischenprodukte aus dem Lebensmittelbereich. Die innovativen Produktlinien sind nach 2010 gegründet worden und wachsen seitdem stetig. 
Die Betriebe selbst sind teilweise jedoch deutlich älter, die Gründungen erfolgten teilweise in den ersten Jahren nach der deutsch-deutschen Wiedervereinigung.

Es wurde ein exploratives Forschungsdesign gewählt, bei dem die Entwicklung von ausgewählten Kleinstbetrieben kontinuierlich zwischen 2015 und 2019 erhoben wurde. Die GründerInnen der Kleinstbetriebe (Lebensmittelmanufakturen) haben innerhalb dieses Zeitfensters Fördergelder erhalten. Mit Hilfe von leitfadengestützten Interviews (,Innovationsbiographien“, ButzIN \& WidMAIER 2016) wurde die Entwicklungsgeschichte einzelner innovativer Produkte erhoben. Innovationsbiographien ermöglichen die Rekonstruktion des Entstehungsprozesses über mehrere Jahre hinweg. Der zeitlich kurze Abstand (kurze Retrospektive) ermöglicht es dabei, auch die Hindernisse und „Umwege“ im Umsetzungsprozess einzufangen, die häufig in langer Retrospektive „geglättet“ werden - wie aus der Biographieforschung bekannt ist (SCHÜTZE 1983). Themen der Interviews waren die Entwicklung des Produktes, der hiermit verbundene Netzwerkaufbau und lokale und nicht-lokale Kontakte. Die Auswertung der Interviews erfolgt für den hier vorliegenden Text nach der dokumentarischen Methode (BoHNSACK 1999), ein komparativ-rekonstruktives Verfahren mit dem Hindernisse und Schwierigkeiten im Umsetzungsprozess verstanden werden sollen. Insgesamt wurden sieben verschiedene Lebensmittelproduzenten seit 2016 teilweise mehrfach interviewt. Deren Perspektive wird ergänzt durch Interviews mit weiteren Personen, die in den jeweiligen Innovationsprozess involviert waren - beispielsweise Zulieferer, Koopera-tionspartner, Branchenvertreter etc. (Summe Interviews: 14). Ergänzt wird diese regionale Fallstudie durch Hintergrundmaterial über die Betriebe aus den Medien und Veröffentlichungen des Landkreises.

\subsection{Networking von Kleinstbetrieben}

Aus den Innovationsbiographien lässt sich der Aufbau lokaler und überregionaler Kontakte rekonstruieren und die Art der Ressourcen, die aus diesen Netzwerken bezogen werden, begrifflich fassen. Dabei zeigt sich, dass die EntrepreneurInnen etablierte Kontakte in translokale oder globale Netzwerke haben, aus denen sie symbolisches Wissen und mitunter auch technisches Know-how beziehen. Die Ideen für innovative Lebensmittelprodukte stammen beispielsweise mitunter aus diesen Kontakten. Die „Neuartigkeit“ besteht dabei typischerweise darin, dass eine vorhandene Idee in den lokalen Kontext umgesetzt wird. Allen EntrepreneurInnen gelingt es (letztendlich) auch, lokale Ressourcen zu erschließen, um die Umsetzung ihrer Produkte voranzutreiben. Allerdings möchte ich im folgenden Abschnitt das Augenmerk auf die Probleme und Widerstände legen, die im Realisierungsprozess überwunden werden müssen. Einerseits sehe ich hierin tendenziell eine Forschungslücke, da üblicherweise über erfolgreiche Netzwerke und Entrepreneure berichtet wird. (Ausnahmen sind beispiels- 
weise Warlow \& Kasabov (2014)). Andererseits relativieren die Schwierigkeiten und Missverständnisse im Netzwerkaufbau die Bedeutung räumlicher Nähe und regionaler Embeddedness als Grundlage für kooperatives Verhalten.

\subsection{1 „Verstehen" und „Nicht-Verstehen" als Muster des Netzwerkaufbaus}

Bei der Entwicklung der innovativen Ideen für ein Lebensmittelprodukt sind die hier untersuchten Entrepreneure häufig beeinflusst von Wissensbeständen, die sie aus anderen Regionen, Kulturen oder gesellschaftlichen Subgruppen ziehen. So wird beispielsweise handwerklich verarbeitetem Essen, besonderen Gewürzen oder auch Geschmacksrichtungen ein besonderer Wert zugeschrieben. Die kulturelle Bedeutung bzw. der „Wert“ von traditionellen oder handwerklich verarbeiteten Lebensmitteln kann als symbolisches Wissen (AsHeIM 2007; MANNICHE \& LARSEN 2013) beschrieben werden.

Mit diesem symbolischen Wissen um den „Wert“ besonderer oder exotischer Lebensmittel ist häufig auch Wissen über den technischen Herstellungsprozess verknüpft. Je nach Technisierungsgrad beziehen die Entrepreneure ihre Maschinen teilweise von wenigen, hochspezialisierten und europaweit tätigen Anlagenbauern. Diese Maschinen stellen eine Materialisierung sowohl branchenspezifischen als auch überregionalen Wissens dar. Die Besonderheit der Anlage führt teilweise dazu, dass brancheninterne Besichtigungen erfolgen. Über diese Spezialanlagen werden die Entrepreneure somit auch wieder rückgebunden in europaweit spannende branchenspezifische Netzwerke. Während Branche und insbesondere Spezialisierungsgrad die Entrepreneure mit anderen Nischenproduzenten weltweit vernetzen, trennt sie diese Position gleichzeitig von den regional ansässigen Betrieben. Dort, wo es in der Region industrielle Fertigungsanlagen im Lebensmittelbereich gibt, bestand bei einigen Entrepreneuren zunächst das Interesse, zusammenzuarbeiten oder ggf. die chargenweise Herstellung in Auftrag zu geben. Wie oben erwähnt, ließen sich Kooperationen mit regional ansässigen Betrieben nicht oder nicht im gewünschten Umfang realisieren. Die Hindernisse lassen sich als Mangel von verschiedenen Formen von „Nähe“ theoretisch erklären. Zunächst haben die Manufakturen aufgrund ihrer geringen Größe und Verarbeitungsmengen Schwierigkeiten, Kooperationen mit der regional ansässigen ,industriellen“ Landwirtschaft bzw. mit der regionalen Lebensmittelindustrie aufzubauen, da sie zu geringe Mengen nachfragen. Dies lässt sich leicht als fehlende institutionelle Nähe erklären. Zusätzlich - und schwerwiegender - jedoch sind die unterschiedlichen Vorstellungen über die Qualität des Produktes ein weiteres Hindernis für regionale Kooperationen:

„Also wir sind da auch an alle [an die regionale Lebensmittelindustrie, GT] ganz offen rangegangen, haben gesagt, wir versuchen jetzt diese Marke hochzubringen mit diesen nostalgischen regionalen Produkten. [..] Es hindert euch niemand 
daran, am Ende des Tages auf eure [Produkt] auch eine verbesserte Rezeptur und ein schöneres Etikett zu machen und es mitzumachen. [...] Aber da ist ganz wenig/ also wirklich kein Verständnis [...] aber ich weiß nicht, das ist ganz, ganz schwer, das in Worte zu fassen. Es fehlt so ein bisschen so ein unternehmerischer Geist am Ende des Tages oder ein bisschen Fantasie [...]. “VR_1 \#35:30\#

Hier drückt sich vor allem eine inkompatible symbolische Wissensbasis aus. Die Schwierigkeit des/der InformantIn, das Problem, nämlich das „Sich-Nicht-Verstehen“, zu beschreiben, entspricht dem häufig non-verbalen oder konjunktiven (Mannheim 1964) Charakter symbolischen Wissens. Obwohl die Akteure in derselben Branche agieren, gibt es unüberbrückbare Differenzen bezüglich der Beschaffenheit des Produktes.

Zum selben Projekt äußert sich ein weiterer Beteiligter folgendermaßen über die schwierige Kooperation:

„, Weil doch ehrlichweise viele gar nicht begreifen, was wir wollen, dass wir ein Handwerk/ Also das ist ja ein/was ja auch total modern ist, so ein handwerkliches Produkt, crafted Produkt [...]. Und ähm keiner versteht so richtig/ oder WILL nicht verstehen oder ich weiß es nicht, woran es liegt, dass man da wieder eine handwerkliche Sache draus machen könnte. "VR_2 \#11:16\#

Dieses generelle Unverständnis für das Unterfangen - ein „Craftfood“-Produkt herzustellen - lässt sich praxistheoretisch ausleuchten. So besteht die unternehmerische Tätigkeit zunächst aus einer kreativen Idee, die abweichend und neu ist. Die Sinngebungsprozesse mit denen die Herstellung dieses Produkts belegt ist (einen Mehrwert für die Region schaffen, den Produzenten der Rohprodukte eine Existenz bieten, traditionelle Strukturen in der Region rekonstruieren), werden zumindest auf emotionaler Ebene vom Gegenüber nicht nachvollzogen. Daraus folgt zweitens auch ein Nicht-Verstehen der Tätigkeiten, die zur Herstellung dieses Produktes notwendig sind, wie beispielsweise die hochwertigere und aufwendigere Verarbeitung des Produktes. Ähnlich inkompatible Wissensstände gibt es innerhalb einer Branche zwischen großen Betrieben und einer Manufaktur. Obwohl diese Betriebe in einem regionalen Branchennetzwerk verbunden sind, misst der/die EntrepreneurIn diesem Netzwerk lediglich einen geringen Wert bei der Beschaffung neuen Wissens bei:

„Für mich wesentlich spannender wäre ein echter Austausch von Know-how, also wenn Sie sich umschauen in der Biogärtnerszene, auf der Welt passiert so viel, und wir in Deutschland, wir schmoren im eigenen Saft und bewegen uns seit den 70er Jahren nicht mehr weiter, böse gesprochen. Also das hat stellenweise alles immer noch die Hippie-Ansätze [...]. " VR_2 \#29:35\# 
Wichtige Quellen für neuartige Technik und Wissen sind für den/die UnternehmerIn dementsprechend nicht die lokalen Betriebe aus derselben Branche, sondern andere Kleinstbetriebe aus dem Ausland:

„Und da gibt es mittlerweile halt, auch in Amerika gibt es Vorbilder, die funktionieren unglaublich gut, da gibt es Know-how-Börsen, da wird gemeinsam an Geräten gearbeitet, die alle aufso Low-Tech-Ebene anzusiedeln sind, aber unglaublich raffiniert sind. [...]. Es gibt jetzt seit nicht allzu langer Zeit das Internet. Und es gibt YouTube Kanäle, also es gibt so viele Informationsmöglichkeiten, wo haargenau gezeigt wird, wie andere [Produzenten] arbeiten, präziseste Informationen gibt es im Internet, auf YouTube in tollen Kanälen. “VR_2 \#29:35\#

Diese Zitate illustrieren, welche Hürden überwunden werden müssen, damit ein echter Wissenstransfer zwischen Betrieben derselben Branche innerhalb einer Region stattfinden kann. Inwiefern die EntrepreneurInnen vor diesem Hintergrund tatsächlich Schnittstellen von globalen Wissensströmen und regionalen Fertigungspraktiken sein können, ist fraglich.

\subsubsection{Ressourcen sichern und erschließen durch lokale Netzwerke}

Da die EntrepreneurInnen, wie oben geschildert, einer nachhaltigen und ökologischen Wirtschaftsweise verpflichtet sind, hat die regionale Beschaffung der Rohstoffe in Bio-Qualität für sie einen sehr hohen Stellenwert. Aufgrund der besonderen Struktur der regionalen (Land-)Wirtschaft (s.o.) ist der Kontakt bzw. Kooperationsaufbau mit lokalen Landwirten jedoch verhältnismäßig schwierig. Als Lösung wird dann der Anbau der Rohstoffe letztendlich im Netzwerk von den beteiligten LandwirtInnen übernommen. Das folgende Zitat verdeutlicht, wie homogen Netzwerke häufig sind:

„Also erst mal vom Netzwerk her ist es so [...] also man zieht ja auch nur das an was man selber ist. [...] Und wir haben [hier in der Region, GT] nur solche idiotischen, konventionellen Landwirte, die dort alles totspitzen [...] Wir geraten mit dem Bauernverband regelmäßig aneinander." VR_4 \#01:05\#.

Hier spiegelt sich deutlich wieder, dass regionale Zugehörigkeit bzw. räumliche Nähe die eklatant unterschiedlichen Einstellungen (kognitive Nähe) nicht überwinden kann. Vielmehr werden enge Beziehungen zu „Gleichen“ aufgebaut und in diesem Kreis die betriebliche Entwicklung vorangetrieben. Die interviewten EntrepreneurInnen betreiben beispielsweise gemeinsam mehrere Läden und einen Markt oder haben die gemeinsame Nutzung landwirtschaftlicher Flächen organisiert. Die hohe Kooperations- 
bereitschaft untereinander geht allerdings, und wenig überraschend, mit Ausgrenzungsmechanismen gegenüber ,anderen“ einher. So dürfen auf dem selbstorganisierten Markt nur ProduzentInnen hochwertiger „Craft“-Produkte ausstellen:

„Aber wir haben auch schon einige wirklich abgelehnt, wo wir gesagt haben, das Produkt passt nicht zu uns. [..] wir sind professionell, wir wollen keine Hobbyleute, wir wollen nicht die Oma, die die Marmelade kocht und da fünf verschiedenen Glasgrößen hat. "VR_8 \#36:12\#

Deutlich spiegelt sich hier auch die Bedeutung ästhetisch-materieller Dimensionen, die ein „Craft"-Produkt ausmachen, beispielsweise im ansprechenden Design der Verpackung.

Die Abgrenzung gegen ein anderes „außen“ fällt mit einer starken Orientierung nach innen zusammen. Hier kann natürlich nicht von gesicherten kausalen Zusammenhängen ausgegangen werden, aber es ist zumindest denkbar, dass die starke Abgrenzung nach außen die Kooperationsbereitschaft untereinander sowohl erhöht als auch notwendiger macht. Andersherum erlaubt erst die gute Kooperationsbasis intern die starke Abgrenzung nach außen. Hinzu kommt, dass sich ausreichend Lifestyle-EntrepreneurInnen in der Region zusammenfinden. Es besteht also regional eine gewisse „kritische Masse", aus der heraus Kooperationen stattfinden können. Diese wird durch den Förderkontext, der die Vernetzung von (Kleinst-)Betrieben zum Ziel hatte, unterstützt.

„Und wenn ich denen [Kunden, GT] dann erzählt habe, bei uns in der Ecke, im Nachbardorf gibt es eine MüHLE und zwei Dörfer weiter ist das MüHLE. Dann kamen sie [Kunden, GT] noch mal zurück teilweise, ,,jetzt geben Sie noch mal [einen Flyer] mit". Dann wurde es interessant, dann wurde es ein Ausflugsziel. Und das war in HAUPTSTADT eben. Die haben gesagt, wegen einer [Mühle, GT] hätten sie sich nicht auf den Weg gemacht, aber wegen dreien hätten sie sich auf den Weg gemacht."VR_6 \#08:11\#

Dementsprechend wird die regionale Netzwerkbildung begünstigt durch den Entstehungskontext. So sind im Laufe weniger Jahre mehrere kleine Betriebe entstanden, die zielstrebig und professionell Strukturen in der Region aufgebaut haben:

„Dann haben wir gesagt, wir müssen zusammen was unternehmen. Wir müssen zusammen Werbung machen und wir fangen alle gerade erst an, uns auszuprobieren. [...] Und haben dann einen Verein gegründet, [...]. Der eine hat den gekannt, der nächste hat den gekannt. Auf einmal saßen 14 Leute in dem Wohnzimmer und haben gesagt, also die gleichen Interessenslagen. “VR_8 \#41:13\# 


\subsection{Diskussion und Schlussfolgerungen}

Die Fallstudie zu einer Gruppe von sogenannten „Lifestyle-EntrepreneurInnen“ in einer ländlich-peripheren aber stark touristischen Region hat gezeigt, dass die Akteure globales Wissen und lokale Ressourcen kombinieren. Die GründerInnen haben ein Interesse daran, breite lokale Kooperationen aufzubauen. Dies ist u.a. auch in ihrer nachhaltigen Wirtschaftsweise und dem Wunsch der regionalen Beschaffung begründet. Allerdings ist dies häufig aufgrund unterschiedlicher Produktionsweisen, aber auch aufgrund unterschiedlicher Einstellungen (fehlende kognitive Nähe oder geteiltes symbolisches Wissen) nicht möglich. Die weitreichenden überregionalen Kontakte, die die EntrepreneurInnen sowohl mitbringen als auch sukzessiv aufbauen, werden genutzt, um symbolisches Wissen in die Region zu holen. Für die Entwicklung der Manufakturen ist der Ausbau lokaler Kooperationen jedoch entscheidend. Durch das Vorhandensein einer „kritischen Masse“ von gleichgesinnten GründerInnen wird eine enge Kooperation untereinander möglich.

Die Ergebnisse dieser explorativen Untersuchung sind nur sehr bedingt auf das Netzwerk- und Innovationsverhalten von EntrepreneurInnen in ländlichen Räumen allgemein übertragbar, da es sich einerseits um eine sehr spezifische Gruppe handelt, die andererseits in einem ländlichen Raum mit ungewöhnlich hoher touristischer Attraktivität und dementsprechenden Zuzugs- und auch Selbstständigenzahlen angesiedelt sind. Der Tourismus trägt dazu bei, dass die kleinen Manufakturen einen „Anschub“ erhalten. Die Spezifik der hier untersuchten Konstellation erlaubt, Aussagen über die Rolle räumlicher Nähe zu treffen. Unter den hier skizzierten Bedingungen reicht räumliche Nähe allein weder aus, um lokale oder regionale Kooperationen zu stiften, noch um fehlende institutionelle Nähe (inkompatible Verarbeitungsmengen) und kognitive Nähe (inkompatible symbolische Wissensbasis) zu überbrücken. Vielmehr zeigt sich, dass Kooperationen primär zwischen „Gleichen“, nämlich nachhaltigkeitsorientierten Lifestyle-EntrepreneurInnen, stattfinden. Die räumliche Häufung dieser „Gleichen“ befördert jedoch deren Kooperationsmöglichkeiten (Austausch von Ressourcen, gemeinsame Verkaufsund Lagerflächen, Maschinennutzung etc.).

Basierend auf der Fallstudie zeigt sich, dass ein Wissenstransfer insbesondere von innovativen Ideen, wie sie aus Subgruppen oder hochspezialisierten Bereichen kommen, in die jeweiligen Branchen kaum stattfindet. Die EntrepreneurInnen sind daher eher keine Schnittstelle zwischen globalen Wissensströmen und regionalen Umsetzungsstrukturen. Damit unterstreichen die Ergebnisse das Problem, dass gerade strukturell schwache Regionen geringe Absorptionsfähigkeiten für neues Wissen haben (OuGHTON et al. 2002). Das Interesse oder den Experimentiergeist regional etablierter Branchen zu stärken, stellt dabei eine deutlich größere Herausforderung für die Regionalentwicklung dar als der Zugang zu Wissen. Insbesondere symbolisches Wissen hat sich in dieser Fallstudie als leicht und preiswert über ICT verfügbar herausgestellt. 


\section{Bibliographie}

Akgün A.A., Baycan-Levent T., Nijkamp P., Poot J. (2011), Roles of Local and Newcomer Entrepreneurs in Rural Development: A Comparative Meta-Analytic Study. Regional Studies 45(9), pp. 1207-23.

Anderson A.R. (2000), Paradox in the Periphery: An Entrepreneurial Reconstruction? Entrepreneurship \& Regional Development 12(2), pp. 91-109.

Asheim B. (2007), Differentiated Knowledge Bases and Varieties of Regional Innovation Systems. Innovation: The European Journal of Social Science Research 20(3), pp. 223-41.

Atterton J. (2016), Invigorating the New Rural Economy - Entrepreneurship and Innovation. In: M. Shucкsmith M. \& Brown D.L. (eds.), Routledge International Handbook of Rural Studies. London, Routledge, pp.165-180.

Buørkhaug H., Kvam G.-T. (2011), Local Small-Scale Food Enterprises: Ambitions and Initiatives for Achieving Business Growth Among Male and Female Owners and Managers. Ager Journal of Depopulation and Rural Development Studies 11, pp. 29-55.

BoHnsack R. (1999), Dokumentarische Methode. In: BoHnSACK R. (ed.). Rekonstruktive Sozialforschung. Wiesbaden, VS Verlag für Sozialwissenschaften, pp. 34-80.

Boschma R. (2005), Proximity and Innovation: A Critical Assessment. Regional Studies 39, pp. 61-74.

Boschma R., Frenken K. (2010), The Spatial Evolution of Innovation Networks. A Proximity Perspective. In: Boschma R., Martin R. (eds.), The Handbook of Evolutionary Economic Geography. Cheltenham, Edwar-Elgar, pp. 120-35.

Bosworth G., Атterton J. (2012), Entrepreneurial In-Migration and Neoendogenous Rural Development: Entrepreneurial In-Migration. Rural Sociology 77(2), pp. 254-79.

Butzin A., Widmaier B. (2016), Exploring Territorial Knowledge Dynamics through Innovation Biographies. Regional Studies 50, pp. 220-232.

Bürcher S., MaYer H. (2018), Are There Differences in Social Capital Related to Corporate Regional Engagement in Dynamic and Less Dynamic Non-Core Regions? European Planning Studies 26(2), pp. 342-64.

Dubois A. (2016), Transnationalising Entrepreneurship in a Peripheral Region - The Translocal Embeddedness Paradigm. Journal of Rural Studies 46, pp. 1-11.

Huggins R., Johnston A. (2009), Knowledge Networks in an Uncompetitive Region: SME Innovation and Growth. Growth and Change 40, pp. 227-259.

KALANTARIDIS C., BiKa Z. (2006), In-Migrant Entrepreneurship in Rural England: Beyond Local Embeddedness. Entrepreneurship and Regional Development 18(2), pp. 109-31.

KNEAfSEy M., Ilbery B., Jenkins T. (2001), Exploring the Dimensions of Culture Economies in Rural West Wales. Sociologia Ruralis 41(3), pp. 296-310.

KorsgaArd S., Müller S., Wittorff TANvig H. (2015a), Rural Entrepreneurship or Entrepreneurship in the Rural - between Place and Space. International Journal of Entrepreneurial Behavior \& Research 21(1), pp. 5-26.

Korsgaard S., Ferguson R., Gaddefors J. (2015b), The Best of Both Worlds: How Rural Entrepreneurs Use Placial Embeddedness and Strategic Networks to Create Opportunities. Entrepreneurship \& Regional Development 27(9-10), pp. 574-98. 
Laschewski L., Phillipson J., Gorton M. (2002), The Facilitation and Formalisation of Small Business Networks: Evidence from the North East of England. Environment and Planning C: Government and Policy 20, pp. 375-391.

Mannheim K. (1964) Das Problem einer Soziologie des Wissens. In: Wolff K.H. (ed.). Karl Mannheim - Wissenssoziologie. Auswahl aus dem Werk. Berlin und Neuwied, Luchterhand, pp. 308-387.

Manniche J., Larsen K.T. (2013), Experience Staging and Symbolic Knowledge: The Case of Bornholm Culinary Products. European Urban and Regional Studies 20(4), pp. 401-16.

MüLler S., KorsgaARD S. (2018), Resources and Bridging: The Role of Spatial Context in Rural Entrepreneurship. Entrepreneurship \& Regional Development 30, pp. 224-255.

Oughton Ch., Landabaso M., Morgan K. (2002), The Regional Innovation Paradox: Innovation Policy and Industrial Policy”. Journal of Technology Transfer 27, pp. 97-110.

RaY Ch. (1999), Endogenous Development in the Era of Reflexive Modernity. Journal of Rural Studies 15(3), pp. 257-67.

Rodríguez-Pose A., Fitjar R.D. (2013), Buzz, Archipelago Economies and the Future of Intermediate and Peripheral Areas in a Spiky World. European Planning Studies 21, pp. 355372.

Schütze F. (1983), Prozessstrukturen des Lebenslaufs. In: Matthes J. (ed.), Biographie in handlungswissenschaftlicher Perspektive. Nürnberg, Verl. d. Nürnberger Forschungsvereinigung, pp. 67-156.

Siebert R., LASCHEwski L. (2010), Creating a Tradition that we never had: Local Food and Local Knowledge in the Northeast of Germany. In: FonTE M., PAPAdopoulos A.G. (eds.). Naming Food after Places: Food Relocalisation and Knowledge Dynamics in Rural Development. Farnham, Ashgate, pp. 61-75.

Tregear A. (2003), Market orientation and the Craftsperson. European Journal of Marketing 37, pp. 1621-1635.

Tregear A. (2005), Lifestyle, Growth, or Community Involvement? The Balance of Goals of UK Artisan Food Producers. Entrepreneurship \& Regional Development 17, pp. 1-15.

Warlow A., Kasabov E. (2014), Re-thinking Rural Conflict, Cooperation Difficulties and Failure: The Case of Rural Cooperatives. In: Kasabov E. (eds.). Rural Cooperation in Europe: In Search of the 'Relational Rurals'. London, Palgrave Macmillan UK, pp. 266-282. 